\title{
Research on Sparse Targets Detection Methods Based on GLRT in Non-Gaussian Clutter
}

\author{
Gu Xinfeng ${ }^{1, ~ *, ~ Y a n ~ S h u q i a n g ~}{ }^{1}$, Hao Xiaolin², Huang Kun ${ }^{1}$ \\ ${ }^{1}$ China Satellite Maritime Tracking \& Control Department, Jiangyin, China \\ ${ }^{2}$ Yantai Electricity and Economy Technical Institute, Yantai, China
}

\author{
Email address: \\ y6ckgxf@126.com (Gu Xinfeng) \\ ${ }^{*}$ Corresponding author
}

\section{To cite this article:}

Gu Xinfeng. Research on Sparse Targets Detection Methods Based on GLRT in Non-Gaussian Clutter. Science Discovery. Vol. 5, No. 1, 2017, pp. 25-32. doi: 10.11648/j.sd.20170501.15

Received: February 21, 2017; Accepted: March 22, 2017; Published: March 31, 2017

\begin{abstract}
For the problem of detecting range-spread target with spare scatterers in non-Gaussian clutter modeled as spherically invariant random vector(SIRV). Firstly, it is assumed that the number of the target scatterers is known and a generalized likelihood ratio test detector based on scatterers number (SN-GLRT) is proposed. Then a sparse scatterers target detector based on GLRT (SST-GLRT) is proposed for unknowing the number of scatterers. The detection statistic of the SSR-GLRT is the weighted sum of the detection statistic of the SN-GLRT. The SSD-SST-GLRT and the NSSD-SST-GLRT are proposed based on the density of the scatterers. The analytical expression relating false alarm probability to detection threshold is deduced and the CFAR property of the SSD-SST-GLRT and the NSSD-SST-GLRT is proved. The results show that the detection performance of NSSD-SST-GLRT is better than the NSDD-GLRT. The detection performance of the SSD-SST-GLRT is better than the SDD-GLRT when the number of scatterers is known. The robustness of the SSD-SST-GLRT is better than the MSDD when the number of scatterers is unknown.
\end{abstract}

Keywords: Non-Gaussian Clutter, Range-Spread Target, Constant False Alarm Rate, Detection

\section{非高斯杂波背景中稀疏距离扩展目标检测方法研究}

顾新锋 ${ }^{1 *}$, 严树强 ${ }^{1}$, 郝晓琳 ${ }^{2}$, 黄坤 ${ }^{1}$

${ }^{1}$ 中国卫星海上测控部, 江阴, 中国

烟台电力经济技术研究所, 烟台, 中国

邮箱

y6ckgxf@126. com（顾新锋）

摘要：针对采用球不变随机向量 (SIRV) 建模的非高斯杂波背景中稀疏扩展距离扩展目标检测问题, 先假设散射个数已 知, 利用广义似然比检验 (GLRT) 原理, 获得了基于散射点数目的GLRT (scatterer number GLRT, SN-GLRT) 检测器。 然后, 针对散射点个数无法先验确定的情况, 提出了基于GLRT的稀疏散射点目标 (sparse scatterers target GLRT, SST-GLRT) 检测器。SST-GLRT 检测统计量是 SN-GLRT 检测统计量的加权求和, 根据散射点密度信息, 分别设计了 SSD-SST-GLRT检测器和NSSD-SST-GLRT检测器, 并推导了其虚警概率与检测门限的解析表达式, 证明了 SSD-SST-GLRT 和NSSD-SST-GLRT的CFAR特性。仿真结果表明, 对于稀疏散射点目标, NSSD-SST-GLRT不需要已知散射点数目的先验信 息, 具有较好的鲁棒性, 并且检测性能优于NSDD-GLRT, 而当散射点个数已知时, SSD-SST-GLRT检测性能优于SDD-GLRT, 散射点个数估计失配时, SSD-SST-GLRT的鲁棒性优于MSDD。 
关键词: 非高斯杂波, 距离扩展目标, 恒虚警率, 检测

\section{1. 引言}

对于低分辨率雷达, 由于雷达的距离分辨率远大于目 标长度, 目标回波表现为单一散射点形式 [1], 关于点目 标的检测问题已经进行了详细的讨论 [1-3]。而脉冲压缩 技术的使用, 使得雷达具有较高的分辨率, 目标回波在雷 达径向上的多个强散射点分布在不同的距离单元中, 形成 距离扩展目标 $[4,5]$ 。提高雷达分辨率并采用有效的方法 可以极大的提高目标检测概率 [6], 但如果仍采用传统点 目标的检测方法, 由于部分目标能量泄漏到参考单元中, 检测性能将大大下降, 甚至完全失效 [7]。文献 [8-10]研 究了高斯背景中扩展目标的检测方法, 然而, 随着雷达分 辨率的提高, 雷达会接受到类似于目标的尖峰, 这种尖峰 可以用球不变随机向量 (spherically invariant random vector, SIRV) 来建模 $[11,12]$, 文献 $[13]$ 利用广义似然比 检验 (generalized likelihood ratio test, GLRT) 的方 法得到了SIRV杂波背景下不依赖于散射点密度的GLRT检 测 器 (non-scatterer density dependent- GLRT, NSDD-GLRT)。NSDD-GLRT是一种被检测距离窗内各距离单 元回波能量的非相干积累检测器, 在实际应用时, 一方面, 由于不同的目标所占距离单元数不同, 为了能够使目标完 全包含在某个被检测距离窗内, 通常需要使窗口长度远大 于目标所占距离单元数; 另一方面, 由于目标的闪炼, 目 标所占距离单元中只有部分单元存在较强的回波, 称之为 强散射点。对于这种稀疏散射点目标, NSDD-GLRT由于积 累了不含目标的距离单元回波, 会出现一定的检测性能损 失，这种损失称为 “坍塌损失” $[14,15]$ 。为了提高检测 器对这种稀疏散射点目标的检测性能, 文献[13]利用散射 点的密度信息, 进一步提出了 SDD-GLRT (scatterer density dependent-GLRT) 检测器。文献 [15]利用目标散 射点的个数信息, 提出了检测性能更好的修正GLRT检测器 (Modified SDD, MSDD), 但当散射点个数未知, 估计值小 于实际值时, MSDD会出现严重的信杂比损失。

针对SIRV建模的非高斯杂波背景下检测稀疏散射点 的扩展目标问题, 本文首先假设目标强散射点的位置信息 已知, 采用GLRT方法得到基于位置信息的GLRT检测器 (scatterer location-GLRT, SL-GLRT), 然后, 采用门限 法估计目标强散射点的位置信息, 再将估计的位置信息代 替SL-GLRT中的位置信息, 得到具有较好鲁棒性的双门限 恒虚警率检测器 (double threshold - constant false alarm ratio, DT-CFAR)。

\section{2. 问题描述}

假设数据从 $N$ 个阵元得到, 需解决跨过连续的 $K$ 个距离 单元检测一个目标存在与否的问题, 其中, $K$ 称为检测窗 口长度。由于目标所占距离单元个数未知, 通常设置的窗 口长度 $K$ 要远大于被检测目标所占距离单元数, 因此, 在 被检测的 $K$ 个距离单元中, 只有部分距离单元存在目标强
散射点回波。令 $\Theta_{K}=\{1,2 \ldots, K\}$ 表示被检测单元的集合， $\Theta_{h}$ 表示目标强散射点所在距离单元的集合, 其中, $h$ 为集 合 $\Theta_{h}$ 的势, 表示目标强散射点个数。假设目标完全包含 在 $K$ 个距离单元内, 则 $\Theta_{h} \in \Theta_{K}$ 。考虑杂波为主的干扰环境, 忽略内部噪声的影响。所要解决的检测问题可表示为:

$$
\begin{aligned}
& \mathrm{H}_{0}: \mathrm{z}_{t}=\mathrm{c}_{t}, \quad t \in \Theta_{K} \\
& \mathrm{H}_{1}: \begin{cases}\mathrm{z}_{t}=\alpha_{t} \mathrm{p}+\mathrm{c}_{t}, & t \in \Theta_{h} \\
\mathrm{z}_{t}=\mathrm{c}_{t}, & t \in \Theta_{K}-\Theta_{h}\end{cases}
\end{aligned}
$$

式中, $\mathrm{H}_{0}$ 表示假设目标不存在, $\mathrm{H}_{1}$ 表示假设目标存 在, $\mathrm{z}_{t}$ 表示第 $t$ 个距离单元总的回波, $\mathrm{c}_{t}$ 表示第 $t$ 个距离单 元的杂波回波, $p$ 表示已知的单位导向矢量, 即 $p^{\mathrm{H}} p=1$ （符 号 “ $(\cdot)^{\mathrm{H}}$ ” 表示共轭转置); 标量 $\alpha$ 是反映目标和信道影 响的未知确定性参数。当阵元为线性均匀阵时, $\mathrm{p}=\left(1, \mathrm{e}^{\mathrm{j} \varphi}, \mathrm{e}^{\mathrm{j} 2 \varphi}, \ldots, \mathrm{e}^{\mathrm{j}(N-1) \varphi}\right)^{\mathrm{T}} / \sqrt{N}$ (其中 $\varphi$ 为常数, 表示各 阵元之间的相位差, 符号 “ $(\cdot)^{\mathrm{T}}$ ” 表示转置)。

杂波回波用SIRV分布来建模, 则杂波向量 $c_{t}$ 可以表示 成

$$
\mathrm{c}_{t}=\sqrt{\tau_{t}} \cdot \eta_{t}, t \in \Theta_{K}
$$

式中, $\eta_{t}=\left(\eta_{t}(1), \quad \eta_{t}(2), \cdots, \quad \eta_{t}(K)\right)^{\mathrm{T}}, \quad \eta_{t}(n)$, $n=1,2, \cdots, N$ 是独立同分布均值为零方差为 1 的复圆高斯随 机变量; 纹理分量 $\tau_{t}$ 是服从分布 $f_{\tau}$ 的非负随机变量, 用 来描述不同距离单元间杂波功率水平的起伏。根据SIRV 模型可知, $\eta_{t}$ 和 $\tau_{t}$ 是相互独立的, 且 $c_{t}$ 的协方差矩阵 $\mathrm{M}$ 表 示为

$$
\mathrm{M}=\mathrm{E}\left\{\mathrm{c}_{t} \mathrm{c}_{t}^{\mathrm{H}}\right\}=\tau_{t} \Sigma, t \in \Theta_{K}
$$

式中, $\Sigma=E\left\{\eta_{t} \eta_{t}^{\mathrm{H}}\right\}$ 为对角元素都为 1 的正定的 Hermitian矩阵, 通常称之为杂波归一化协方差矩阵或杂 波协方差矩阵结构。

\section{GLRT检测器设计}

假设在 $K$ 个距离单元中, 各距离单元杂波相互独立, 则在式 (1) 给出的每个假设下 $\mathrm{z}=\left[\mathrm{z}_{1}^{\mathrm{T}}, \ldots, \mathrm{z}_{K}^{\mathrm{T}}\right]^{\mathrm{T}}$ 的概率密度 函数可以分别表示为

$$
f\left(\mathrm{z} \mid \mathrm{H}_{0}\right)=\prod_{t \in \theta_{K}} \frac{1}{\tau_{t}^{N} \pi^{N} \operatorname{det}(\Sigma)} \exp \left(-\frac{\mathrm{z}_{t}^{\mathrm{H}} \Sigma^{-1} \mathrm{z}_{t}}{\tau_{t}}\right)
$$




$$
\begin{aligned}
& f\left(\mathrm{z} \mid \mathrm{H}_{1}\right)=\prod_{t \in \Theta_{K}-\theta_{h}} \frac{1}{\tau_{t}^{N} \pi^{N} \operatorname{det}(\Sigma)} \exp \left(-\frac{\mathrm{z}_{t}^{\mathrm{H}} \Sigma^{-1} \mathrm{z}_{t}}{\tau_{t}}\right) \times \\
& \prod_{t \in \Theta_{h}} \frac{1}{\tau_{t}^{N} \pi^{N} \operatorname{det}(\Sigma)} \exp \left(-\sum_{t \in \Theta_{h}} \frac{\left(\mathrm{z}_{t}-\alpha_{t} \mathrm{p}\right)^{\mathrm{H}} \Sigma^{-1}\left(\mathrm{z}_{t}-\alpha_{t} \mathrm{p}\right)}{\tau_{t}}\right)
\end{aligned}
$$

式中, $\operatorname{det}(\cdot)$ 表示行列式。对于GLRT, 在每个假设下 未知的参数采用最大似然估计得到。然后, 将每个假设下 的估计值代入到式 (4) 和式 (5) 中, 由式 (5) 和式 (4) 给出的 最终表达式的比称为GLRT。这里假设杂波协方差矩阵结构 已知, 则在 $\mathrm{H}_{0}$ 假设下, 未知的参数为杂波纹理分量 $\tau=\left[\tau_{1}, \ldots, \tau_{K}\right]$, 而在 $H_{1}$ 假设下，未知的参数为 $\tau$, $\alpha=\left[\alpha_{r_{1}}, \ldots, \alpha_{r_{h}}\right]$ 和 $\Theta_{h}$, 则GLRT可以表示为

$$
\begin{gathered}
\stackrel{\mathrm{H}_{1}}{>} T \\
< \\
\mathrm{H}_{0}
\end{gathered}
$$

式中,

$$
\lambda=\frac{\max _{\alpha, \tau, \Theta_{h}} f\left(\mathrm{z} \mid \mathrm{H}_{1}\right)}{\max _{\tau} f\left(\mathrm{z} \mid \mathrm{H}_{0}\right)}
$$

是GLRT的检验统计量, $T$ 为检测器门限。

首先, 考虑 $\alpha$ 和 $\tau$ 的估计值。在 $H_{0}$ 假设下, $\tau_{t}$ 的最大 似然估计可以表示为

$$
\hat{\tau}_{0, t}=\frac{\mathrm{z}_{t}^{\mathrm{H}} \Sigma^{-1} \mathrm{z}_{t}}{N}, \quad \underset{t \in \Theta_{K}}{ }
$$

在 $H_{1}$ 假设下, $\tau_{t}$ 的最大似然估计可以表示为

$$
\hat{\tau}_{1, t}=\left\{\begin{array}{l}
\frac{\mathrm{z}_{t}^{\mathrm{H}} \Sigma^{-1} \mathrm{z}_{t}}{N}, t \in \Theta_{K}-\Theta_{h} \\
\frac{\left(\mathrm{z}_{t}-\alpha_{t} \mathrm{p}\right)^{\mathrm{H}} \Sigma^{-1}\left(\mathrm{z}_{t}-\alpha_{t} \mathrm{p}\right)}{N}, t \in \Theta_{h}
\end{array}\right.
$$

$\alpha_{t}$ 的最大似然估计为

$$
\alpha_{t}=\frac{\mathrm{p}^{\mathrm{H}} \Sigma^{-1} \mathrm{z}_{t}}{\mathrm{p}^{\mathrm{H}} \Sigma^{-1} \mathrm{p}}, t \in \Theta_{h}
$$

将式 (8) 式 (10) 代入到式 (7) 经化解得

$$
\lambda=\max _{\Theta_{h}} \prod_{t \in \Theta_{h}}\left[1-w_{t}\right]^{-N}
$$

式中,

$$
w_{t}=\frac{\left|\mathrm{p}^{\mathrm{H}} \Sigma^{-1} \mathrm{z}_{t}\right|^{2}}{\left(\mathrm{p}^{\mathrm{H}} \Sigma^{-1} \mathrm{p}\right)\left(\mathrm{z}_{t}^{\mathrm{H}} \Sigma^{-1} \mathrm{z}_{t}\right)} 。
$$

注意到， $w_{t}$ 是复合高斯杂波中归一化匹配滤波器 (normalized matched filter, NMF) 的检测统计量。 $w_{t}$ 可 以看成是 $\mathrm{p}$ 和 $\mathrm{z}_{t}$ 经过白化和归一化后的内积, 满足 $0 \leq w_{t}<1$ 。

由于 $\Theta_{h}$ 不仅包含了目标散射点的位置信息, 也包含 了散射点的个数信息 $h$ 。当散射点的位置信息已知时, $\Theta_{h}$ 为已知量, 不需要估计。因此, 考虑散射点位置信息未知, 分别从 $h$ 未知和 $h$ 已知两种情况讨论 $\Theta_{h}$ 的估计及GLRT统计 量。

\section{1) $h$ 未知}

当 $h$ 未知时, 由于 $\left[1-w_{t}\right]^{-N} \geq 1$, 因此, $h$ 的估计值为 $h$ 能取得的最大值, 即 $\hat{h}=K, \hat{\Theta}_{h}=\Theta_{K}$, 式(11) 可以表示 为

$$
\lambda=\prod_{t \in \Theta_{K}}\left[1-w_{t}\right]^{-N}
$$

由于检测统计量经过单调递增函数变换后可得到等 效的检测统计量, 因此, 当 $h$ 未知时, GLRT的等效检测统 计量可以表示为

$$
\lambda_{\mathrm{GLRT}}=-2(N-1) \sum_{t \in \Theta_{K}} \ln \left(1-w_{t}\right)
$$

注意到, $\lambda_{\mathrm{GLRT}}$ 与文献 [7]中的NSDD-GLRT是一致的。 这表明, 当 $h$ 未知时, 稀疏散射点目标的GLRT与假设每个 单元都存在目标散射点的GLRT是等价的。这是因为, 不论 实际的 $h$ 取何值, 其最大似然估计都是 $\hat{h}=K$ 。

2） $h$ 已知

当 $h$ 已知时, 先计算 $w_{t}, t \in \Theta_{K}$, 然后对 $w_{t}$ 从大到小进 行排序。令 $r_{i}$ 表示排序后第 $i$ 个值对应的距离单元编号, 即 $w_{r_{1}} \geq w_{r_{2}} \geq \cdots \geq w_{r_{K}}$ 。则, $\Theta_{h}$ 的最大似然估计可以表示 为

$$
\hat{\Theta}_{h}=\left\{r_{1}, r_{2}, \ldots, r_{h}\right\}
$$

将式(15) 代入式 (11), 再经过单调递增函数变换后, 可得到基于散射点个数 (scatterers number, $\mathrm{SN}$ ) 的等效 GLRT统计量

$$
\lambda_{\mathrm{SN}-\mathrm{GLRT}}(h)=-2(N-1) \sum_{i=1}^{h} \ln \left(1-w_{r_{i}}\right)
$$

对比式 (14) 和式 (16), 不难发现, GLRT利用了所有距 离单元回波进行积累, 而SN-GLRT只利用 $h$ 个距离单元回波 进行积累。当 $h=K$ 时, SN-GLRT退化为GLRT。

GLRT (NSDD-GLRT) 不需要目标散射点个数的先验信息, 但对于稀疏散射点目标时, 由于积累了不含散射点的距离 单元回波, 存在一定的 “圾塌损失”。而SN-GLRT (MGLRT, OS-GLRT) 只利用部分距离单元进行积累, 有效的克服了 “坍塌损失”, 但需要散射点个数的先验信息, 实际应用 
时, 由于目标的大小, 方位角的不同, 以及目标的闪㷧, 很难确定散射点的个数。

借鉴文献 [7]中SDD-GLRT检测器设计思路, 稀疏散射 点目标GLRT (sparse scatterer target-GLRT, SST-GLRT) 检测统计量表示如下

$$
\lambda_{\text {SST-GLRT }}=\sum_{h=1}^{K} p_{h} \lambda_{\text {SN-GLRT }}(h)
$$

式中, $p_{h}$ 表示 $\mathrm{K}$ 个距离单元中散射点个数是 $\mathrm{h}$ 的概率, 并且有 $\sum_{h=1}^{K} p_{h}=1$ 。根据式 (17) 容易看出, $\lambda_{\text {SST-GLRT }}$ 是 SN-GLRT检测统计量 $\lambda_{\text {SN-GLRT }}(h)$ 的加权求和。需要指出的是, 当

$$
p_{h}=\left\{\begin{array}{l}
0, h=1, \ldots, K-1 \\
1, h=K
\end{array}\right.
$$

时, 相当于每个单元都存在目标散射点, SST-GLRT 就退化为GLRT。当

$$
p_{h}=\left\{\begin{array}{l}
0, h \in \Theta_{K}, h \neq m \\
1, h=m
\end{array}\right.
$$

时, 相当于目标散射点个数为 $m$, SST-GLRT退化为散射点 个数为 $m$ 的SN-GLRT。这表明GLRT和SN-GLRT都是SST-GLRT 的特例。

下面考虑另外两种典型的SST-GLRT。一种是假设已知 散射点的密度信息 $\alpha$, 相应的SST-GLRT不妨称之为依赖于 散射点密度的SST-GLRT (SDD-SST-GLRT)，与SDD-GLRT相 似, $p_{h}$ 可以表示为

$$
p_{h}=c_{1}\left(\begin{array}{l}
K \\
h
\end{array}\right)(1-\alpha)^{K-h} \alpha^{h}, h=1, \ldots K
$$

式中, $c_{1}=\left(1-(1-\alpha)^{K}\right)^{-1}$ 。则SDD-SST-GLRT统计量可 以表示为

$$
\lambda_{\text {SDD-SST-GLRT }}=c_{1} \sum_{h=1}^{K}\left(\begin{array}{l}
K \\
h
\end{array}\right)(1-\alpha)^{K-h} \alpha^{h} \sum_{i=1}^{h} u_{r_{i}}
$$

式中, $u_{r_{i}}=-2(N-1) \ln \left(1-w_{r_{i}}\right)$ 。需要指出的是 SSD-SST-GLRT与SSD-GLRT的主要区别在于, SSD-SST-GLRT 利用SN-GLRT统计量的加权求和而SSD-GLRT利用所有可能 的GLRT统计量平均值的加权求和。

另一种是不依赖于散射点密度的SST-GLRT, 不妨称之 为NSDD-SST-GLRT, 即假设 $K$ 个距离单元中, 存在 $1, \ldots, K$ 个散射点的概率相等, 这时,

$$
p_{h}=\frac{1}{K}, h=1, \ldots, K
$$

NSDD-SST-GLRT检测统计量可以表示为

$$
\lambda_{\text {NSDD-SST-GLRT }}=\frac{1}{K} \sum_{h=1}^{K} \sum_{i=1}^{h} u_{r_{i}}
$$

这里NSDD-SST-GLRT和NSDD-GLRT的主要区别在于, NSDD-SST-GLRT 是对 SN-GLRT 统计量求平均值, 而 NSDD-GLRT 只是SN-GLRT在 $h=K$ 时的特例。

\section{4. 虚警概率分析}

由于 SSD-SST-GLRT 和SSD-SST-GLRT 都是 SST-GLRT 的 特例, 不同之处在于加权系数 $p_{h}$ 的取值不同。下面利用 SST-GLRT 统计量分析这两个检测器虚警概率和检测门限 的关系。

令 $u_{t}=-2(N-1) \ln \left(1-w_{t}\right)$, 则 $u_{r_{i}}=-2(N-1) \ln \left(1-w_{r_{i}}\right)$, 并且有 $u_{r_{1}} \geq \cdots \geq u_{r_{K}}$ 。式 (17) 可以重新表示为

$$
\lambda_{\mathrm{SST}-\mathrm{GLRT}}=\sum_{h=1}^{K} p_{h} \sum_{i=1}^{h} u_{r_{i}}=\sum_{i=1}^{K} P_{i} u_{r_{i}}
$$

式中, $P_{i}=\sum_{h=i}^{K} p_{h}$, 其中, $P_{1}=1$ 。式 (3.1) 表明, SST-GLRT统计量可看成是对 $u_{t}$ 的加权求和, 并且较大的 $u_{t}$ 赋予较高的权值, 其中, $u_{t}$ 是每个距离单元NMF统计量 $w_{t}$ 经过单调递增函数变换后的统计量。

在 $\mathrm{H} 0$ 假设下, 文献 $[\mathrm{NSDD}]$ 的分析结果表明, $u_{t}$ 服从自 由度为为 2 的 $\chi^{2}$ 分布 (参数为 2 的指数分布), 其概率密度 函数 (PDF, probability density function) 为:

$$
f_{u_{t}}(x)=\frac{1}{2} \mathrm{e}^{-x / 2}, x \geq 0
$$

由于各距离单元回波 $\mathrm{z}_{t}, t=1,2, \cdots, K$ 是统计独立的, 因此, $u_{t}, t=1,2, \cdots, K$ 之间也相互统计独立, 但经排序以 后所得的有序样本 $u_{r_{1}}, \ldots, u_{r_{K}}$ 之间并非统计独立, 经线性变 换

$$
\left\{\begin{aligned}
v_{1}= & P_{1}\left(u_{r_{1}}-u_{r_{2}}\right) \\
v_{2}= & \left(P_{1}+P_{2}\right)\left(u_{r_{2}}-u_{r_{3}}\right) \\
& \vdots \\
v_{k}= & \left(\sum_{m=1}^{k} P_{m}\right)\left(u_{r_{k}}-u_{r_{k+1}}\right) \\
& \vdots \\
v_{K}= & \left(\sum_{m=1}^{K} P_{m}\right) u_{r_{K}}
\end{aligned}\right.
$$

所得变量 $v_{1}, \cdots, v_{K}$ 是统计独立的随机变量序列。并 且有 


$$
\sum_{k=1}^{K} v_{k}=\sum_{k=1}^{K} P_{k} u_{r_{k}}
$$

式中, $v_{i}$ 的PDF为

$$
f_{v_{k}}(x)=\frac{k}{2 q_{k}} \mathrm{e}^{-k x /\left(2 q_{k}\right)}, \quad x \geq 0
$$

式中, $q_{k}=\sum_{m=1}^{k} P_{m}$ 。

结合式 (3.1) 和式 (3.5) 可得

$$
\lambda_{\mathrm{SST}-\mathrm{GLRT}}=\sum_{k=1}^{K} v_{k}
$$

即SST-GLRT统计量是独立随机变量 $v_{1}, \cdots, v_{K}$ 的和, 因此 $\lambda_{\text {SST-GLRT }}$ 的矩母函数 (monment generating function, MGF) 是 $v_{k}$ 的MGF的积, 即

$$
M_{\lambda_{\text {SST-GLRT }}}(x)=\prod_{k=1}^{K} M_{v_{k}}(x)
$$

式中，

$$
M_{v_{k}}(x)=\frac{1}{1+\left(2 q_{k} / k\right) x}, \quad k=1,2, \ldots, K
$$

是 $v_{k}$ 的MGF。

对 $M_{\lambda_{\text {SST-GLT }}}(x)$ 进行拉普拉斯反变换可得 $\lambda_{\text {SST-GLRT }}$ 的 $\mathrm{PDF}$, 令 $a_{k}=k /\left(2 q_{k}\right)$, 当 $p_{1} \neq 0$ 时（对于SSD-SST-GLRT和 SSD-SST-GLRT, 这一条件是满足的), $\lambda_{\text {SST-GLRT }}$ 的PDF可 以表示为

$$
f_{\lambda_{\text {SST-GLRT }}}(y)=\sum_{k=1}^{K} d_{k} e^{-a_{k} y}
$$

式中,

$$
d_{k}=a_{k} \prod_{m \in \Theta_{k}, m \neq k}\left(1-a_{k} / a_{m}\right)^{-1}
$$

SST-GLRT的虚警概率可以表示为

$$
P_{\mathrm{fa}}=\int_{T}^{\infty} f_{\lambda_{\text {SST-GRT }}}(y) \mathrm{d} y=\sum_{k=1}^{K} c_{k} e^{-a_{k} T}
$$

式中,

$$
\begin{gathered}
c_{k}=\prod_{m \in \Theta_{K}, m \neq k}\left(1-a_{k} / a_{m}\right)^{-1} \\
a_{k}=k\left(2 \sum_{m=1}^{k} \sum_{i=m}^{K} p_{i}\right)^{-1}
\end{gathered}
$$

分别将式 (20) 和式 (22) 代入式 (36) 即可得到 SDD-SST-GLRT和NSDD-SST-GLRT的虚警概率与检测门限的 关系表达式。

由式 (34) 可知, 虚警概率只与检测门限 $T$, 检测窗口 宽度 $K$ 以及选择的加权系数 $p$ 有关，而与杂波的统计参数无 关, 这表明 SDD-SST-GLRT 和 NSDD-SST-GLRT 都是恒虚警 (constant false alam ratio, CFAR) 检测器。式 (34) 还 表明, 虚警概率与 $N$ 无关, 即对于给定的虚警概率和 $K$, 不 同的 $N$ 值对应的检测门限是相同的, 而SDD-GLRT需要根据 不同的 $N$ 值设定不同的检测门限, 并且其检测门限很难计 算得到, 需要采用Monte Carlo方法估计。

图1和图2分别给出了SDD-SST-GLRT和NSDD-SST-GLRT 虚警概率与检测门限的关系曲线。图 1 和图 2 表明, 对于给 定的虚警概率, $K$ 越大, 检测门限也就越大, 这是因为 $K$ 越大, 积累的数据也就越多。图1还表明, SDD-SST-GLRT 的检测门限随着 $\alpha$ 的增大而增大, 这时因为, 增大 $\alpha$ 相当 于增大了 SN-GLRT统计量中较大值的权值。

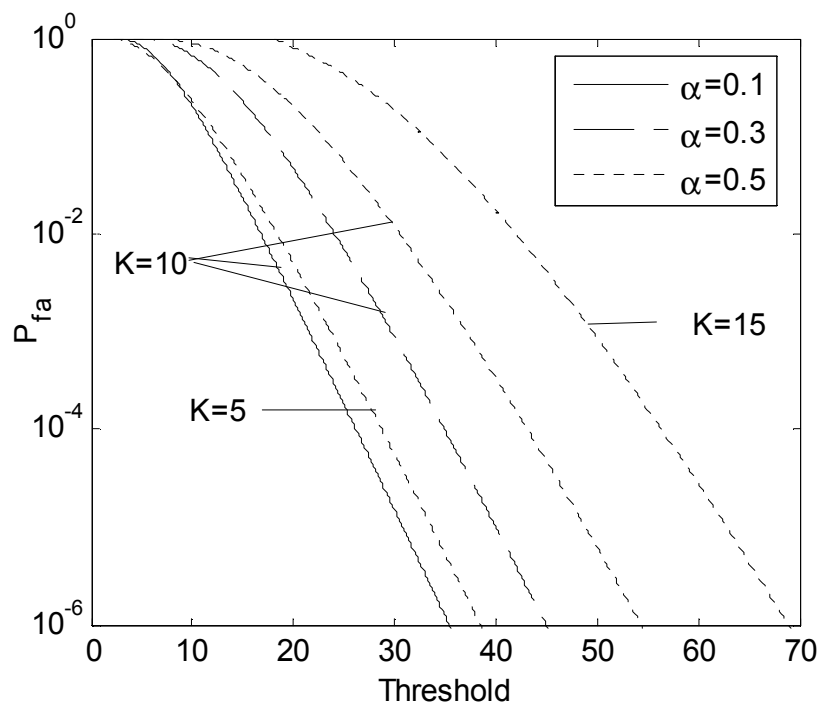

图1 SDD-SST-GLRT虚警概率与检测门限关系曲线。

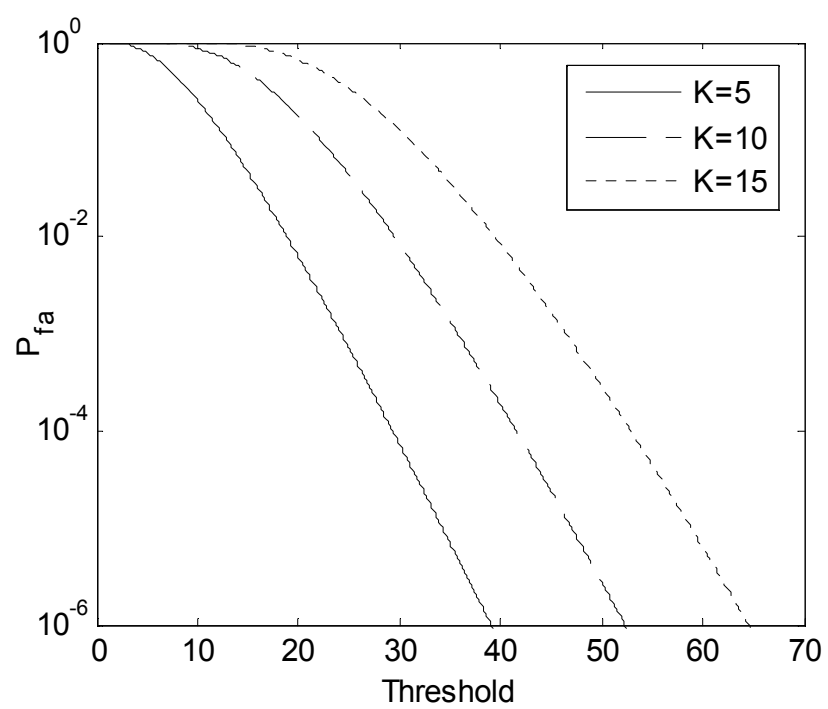

图2 NSDD-SST-GLRT虚警概率与检测门限关系曲线。 


\section{5. 性能分析}

采用Monte Carlos仿真技术对本文方法的性能进行 分析。仿真中, 采用 $\Gamma$ 分布来表示混合分布 $f_{\tau}$

$$
f_{\tau}(\tau)=(L / b)^{L} \tau^{L-1} \mathrm{e}^{-(L / b) \tau} / \Gamma(L), \quad \tau \geq 0
$$

式中 $\Gamma(\cdot)$ 是Gamma函数, $b$ 是均值, $L$ 用来控制与正态 统计量偏离的程度, L越小, $f_{\tau}(\tau)$ 函数的拖尾越长, 杂波 就会出现更多的尖峰。不失一般性, 在仿真中令 $b=1$, 即 把杂波功率归一化为一个定值, 此时的距离单元中杂波的 分布函数等效为 $K$ 分布 ${ }^{[15]}$ :

$$
f_{x}(x)=\frac{2 L}{\Gamma(L)}(x \sqrt{L / 2})^{L-1} \mathrm{~K}_{L-1}(x / \sqrt{2 L})
$$

式中, $K_{L}$ 表示第二类修正的Bessel函数。

假设被检测单元中目标的平均功率为 $\sigma_{s}^{2}$, 杂波平均 功率为 $\sigma_{\mathrm{c}}^{2}$ 。存在目标散射点的距离单元回波采用方差为 $\varepsilon_{t} \sigma_{s}^{2} K$ 独立零均值复圆高斯随机变量建模, 其中, $\varepsilon_{t}$ 表示 距离单元 $t$ 中目标能量占总能量的比列。这里考虑几种典 型的多主散射点目标模型, 如表1所示。

表1 不同目标模型的 $\varepsilon_{t}$ 值。

\begin{tabular}{lllll}
\hline 距离单元 & 1 & 2 & $\cdots$ & $h$ \\
\hline Mode1. 1 & $1 / h$ & $1 / h$ & $1 / h$ & $1 / h$ \\
Mode1. 2 & 0.5 & $0.5 /(\mathrm{h}-1)$ & $0.5 /(\mathrm{h}-1)$ & $0.5 /(\mathrm{h}-1)$ \\
Mode1. 3 & 0.9 & $0.1 /(\mathrm{h}-1)$ & $0.1 /(\mathrm{h}-1)$ & $0.1 /(\mathrm{h}-1)$ \\
\hline
\end{tabular}

信杂比定义为:

$$
\operatorname{SCR}=\left(\mathrm{p}^{\mathrm{H}} \Sigma^{-1} \mathrm{p}\right) \sigma_{\mathrm{s}}^{2} / \sigma_{\mathrm{c}}^{2}
$$

仿真中，假设 $K=15 ， N=2, L=1$ ，虚警概率为 $10^{-3}$ ，检 测门限根据式 (3.11) 计算得到, 检测概率采用Monte Carlo方法计算。

图3 (a) 图 3 (c) 分别针对表1中的三种目标模型分析 SDD-SST-GLRT和NSDD-SST-GLRT的检测性能, 为表示方便, 下面直接用SDD-SST和NSDD-SST表示, 同样, 用MSDD, SDD 和NSDD表示MSDD-GLRT, SDD-GLRT和NSDD-GLRT。其中, $h=3$ 。 图 1(a) 图 1 (c) 表明, 对于不同的多主散射点目标, NSDD-SST 的检测能优于普通的NSDD检测器, 这是因为 NSDD-SST利用了目标稀疏散射点的特性, 对较强的散射点 回波赋予较大的权值，而较弱的散射点或杂波回波赋予较 小的权值, 在一定程度上减小了普通NSDD由于积累乐不含 目标散射点的距离单元回波出现的 “坍塌损失”。在散 射点个数信息已知时, MSDD充分利用这一信息, 进一步增 强了检测器的性能, 而SDD-SST利用了散射点的密度信息 $(\alpha=h / J)$, 具有与MSDD近似的检测性能并且明显优于 $\mathrm{SDD}$ 。

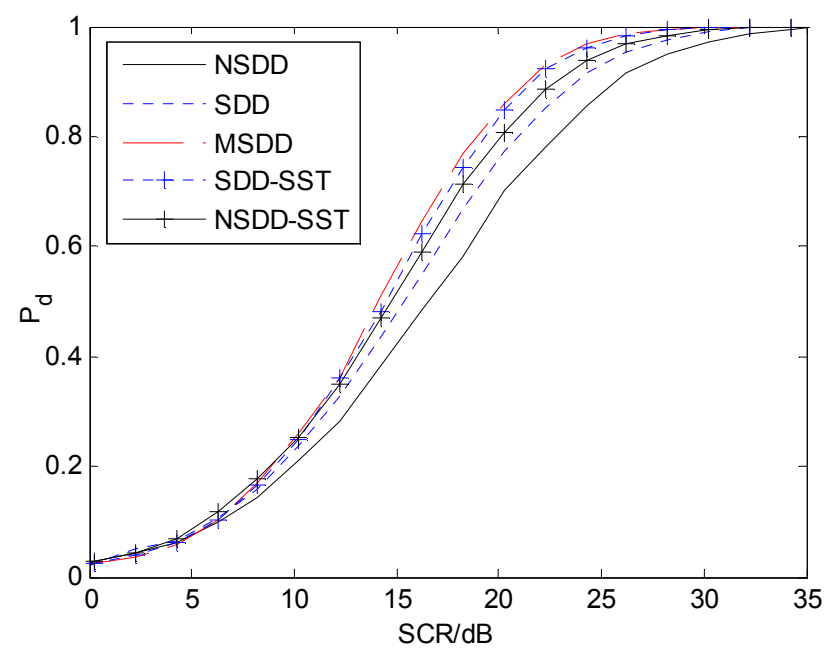

(a) Mode1. 1

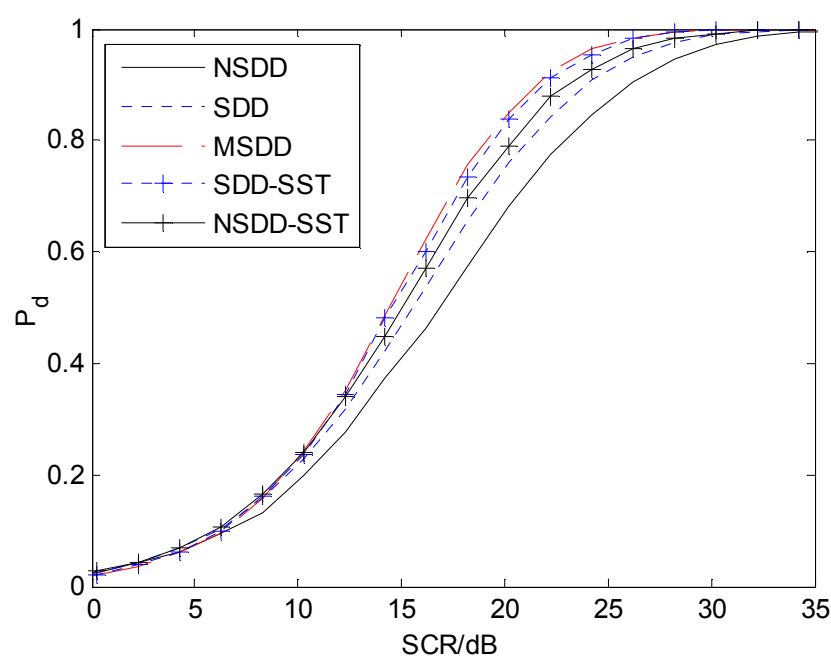

(b) Mode1. 2

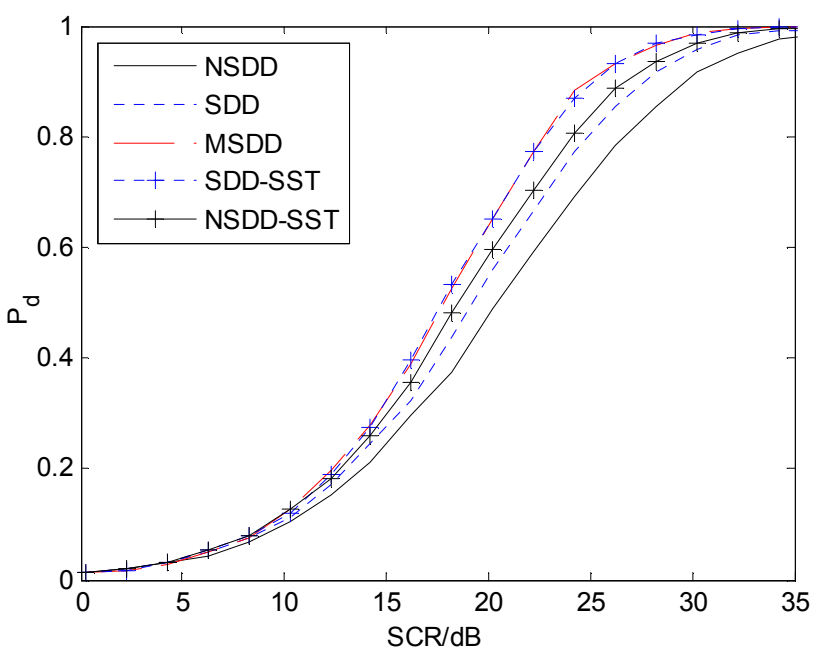

(c) Mode1. 3

图3 检测性能比较, $h=3$ 。

在实际应用时, 通常, 散射点的个数信息是未知的, 需要实现进行估计, 当估计个数与实际个数不一致时, 即 
出现估计失配, 从而影响检测器的性能, 图4 图6 分析了 散射点个数估计失配时检测器的性能。其中, 图4和图5 针对Mode1. 3 分析了MSDD和SDD-SST散射点个数估计失配 时的检测性能。其中, $h=3$ 。图 4 表明, 即使目标 $90 \%$ 的能 量集中于一个散射点时, 当散射点个数估计值为 1 时, MSDD 也会出现严重的性能损失。图5则表明, SDD-SST在散射点 数目估计失配时, 鲁棒性要优于MSDD。这时因为当散射点 个数估计值小于实际值时, MSDD不能有效积累所有目标散 射点回波能量, 而SDD-SST通过一定的权值, 积累了所有 散射点的回波能量。

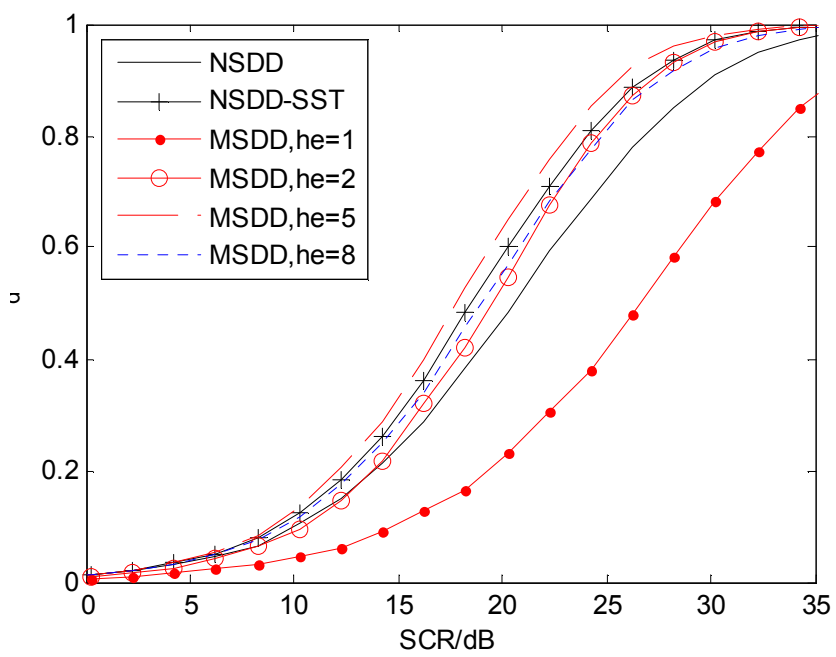

图4 MSDD失配性能分析, $h=3$ 。

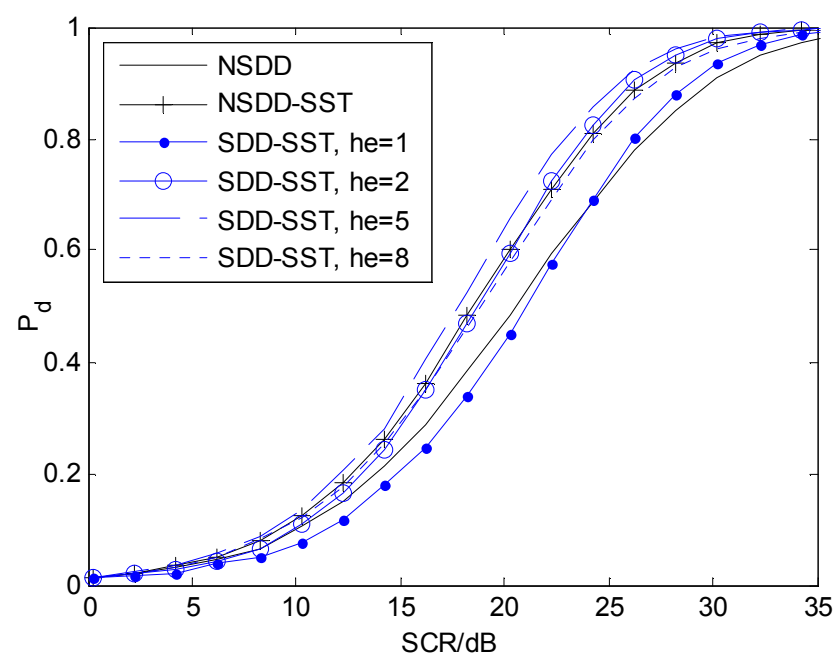

图5 SDD-SST失配性能分析, $h=3$ 。

图6进一步针对Mode1. 1 分析了信杂比为 $20 \mathrm{~dB}$, 散射 点个数为 3 时, 各检测器检测概率与散射点个数估计的关 系曲线, 其中, NSDD和NSDD-SST不需要估计散射点个数, 每次估计进行 5000 次仿真, 求平均检测概率。图6表明, 当目标散射点个数估计值小于实际值时, MSDD和SDD-SST 的检测概率下降都比较严重, 但SDD-SST的检测概率要高 于MSDD。当散射点个数估计值大于实际值时, SDD-SST和 MSDD的检测概率都随之估计值的增大而减小, 并且检测概 率都要大于 SDD, 当 $h_{\mathrm{e}}=K$ 时, 都等价为 NSDD。NSDD 和
NSDD-SST 都不受散射点个数估计值的影响, 并且, NSDD-SST的检测概率大于NSDD, 这是因为, NSDD对所有回 波赋予相同的权值, 而NSDD-SST利用了目标具有稀疏散射 点的特性, 对较强的散射点赋予较大的权值, 而较弱的散 射点或杂波回波赋予较小的权值, 减小了NSDD的 “坍塌损 失”，从而增强了稀疏散射点目标的检测概率。图6还表 明, NSDD-SST的检测性能优于SDD。

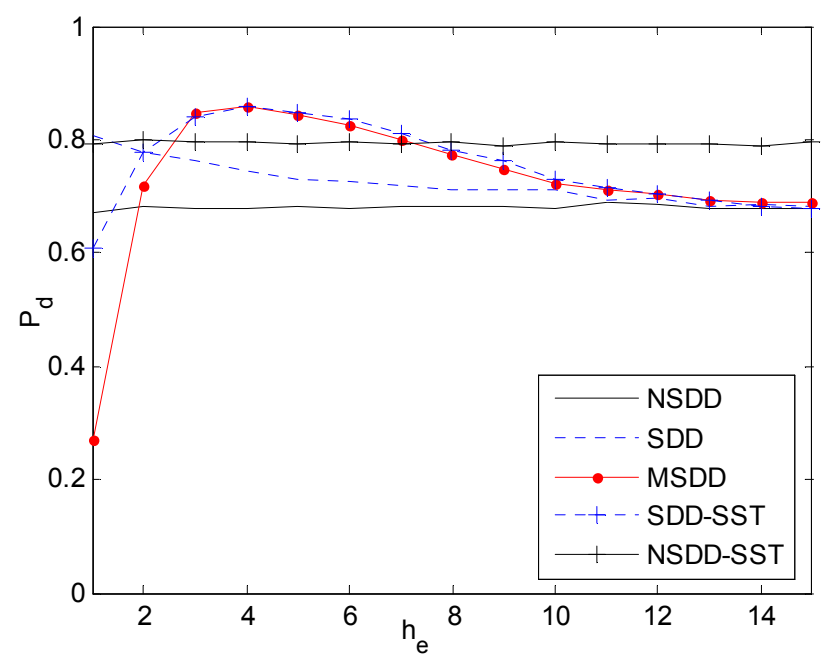

图6 失配性能比较, $h=3, \mathrm{SCR}=20 \mathrm{~dB}$ 。

\section{6. 结论}

本文研究了SIRV建模的复合高斯杂波背景下的稀疏 距离扩展目标检测问题。针对被检测窗口长度远大于目标 所占距离单元数时, 利用所有距离单元回波进行积累, 因 大量积累了不含目标散射点距离单元回波能量, 从而出现 一定的 “坍塌损失”。针对这种情况, 即被检测单元中目 标具有稀疏散射点的情况, 首先, 假设目标散射点个数已 知, 利用GLRT原理, 提出了 SN-GLRT, 分析表明, SN-GLRT 等价为文献 [9] 中的MSDD检测器。然后, 针对散射点个数 无法先验确定的情况, 提出了基于GLRT的稀疏散射点目标 (SST-GLRT) 检测器. SST-GLRT检测统计量是SN-GLRT检测 统计量的加权求和, 并给出了两个SST-GLRT的具体实例 SSD-SST-GLRT和NSSD-SST-GLRT。推导了SSD-SST-GLRT和 NSSD-SST-GLRT虚警概率与检测门限关系的解析表达式。 SSD-SST-GLRT利用了散射点密度的先验信息, 对可能出现 散射点个数概率的大小赋予 SN-GLRT统计量不同的权值, 而NSSD-SST-GLRT针对散射点先验信息完全未知情况下, 对所有SN-GLRT统计量进行等权值相加。结果表明, 在已 知散射点个数时, MSDD 具有最优的检测性能, 而 SSD-SST-GLRT的检测性能接近于MSDD。但散射点个数信息 未知时, NSSD-SST-GLRT不需要估计散射点个数, 并且检 测性优于NSDD, 而SSD-SST-GLRT和MSDD的检测性能受散射 点个数估计值的影响, 并且SSD-SST-GLRT的鲁棒性优于 MSDD。 


\section{参考文献}

[1] 何友, 关键, 彭应宁, 等. 雷达自动检测与恒虚警处理 [M], 北京, 清华大学出版社, 1999。

[2] Kelly E J. An adaptive detection algorithm [J]. IEEE Trans-actions on Aerospace and Electronic Systems, 1986, $22(1)$ : 115-127.

[3] 何友, 关键, 孟祥伟, 等. 雷达自动检测和CFAR处理方法综述 [J]．系统工程与电子技术，2001，23(1)：9-14,85。

[4] Shui P L, Liu H W, Bao Z. Range-spread target detection based on cross time-frequency distribution reatures of two adjacent received signals [J]. IEEE Transactions on Signal Processing, 2009, $57(10)$ : 3733-3745.

[5] 简涛, 何友, 苏峰, 等. 高距离分辨率雷达目标检测研究现状 与进展 [J]. 宇航学报, 2010，31 (12)：2623-2628. [Jian Tao, He You, Su Feng, et al. Overview of high range resolution radar target detection $[\mathrm{J}]$. Journal of Astronautics, 2010, 31(12): 2623-2628.]。

[6] Hughes P K. A high-resolution radar detection strategy [J]. IEEE Transactions on Aerospace and Electronic Systems, 1983, (19): 663-667.

[7] Gerlach K. Spatially distributed target detection in non-Gaussian clutter [J]. IEEE Transactions on Aerospace and Electronic Systems, 1999, $35(3)$ : 926-934.

[8] Xu S, Shui P, Yan X. CFAR detection of range-spread target in white Gaussian noise using waveform entropy [J]. Electronics Letters, 2010, 46(9) : 647-649.

[9] Bandiera F, Orlando, D, Ricci G. CFAR detection strategies for distributed targets under conic constraints [J]. IEEE Transactions on Signal Processing, 2009, 57 (9): 3305-3316.

[10] Shui P L, Xu S W, Liu H W. Range-spread target detection using consecutive HRRPs [J]. IEEE Transactions on Aer-ospace and Electronic Systems, 2011, $47(1):$ 647-665.

[11] He Y, Jian T, Su F, et al. Two adaptive detectors for range-spread targets in non-Gaussian clutter. Sci China Ser F-Inf Sci, 2011, 54: $386-395$.

[12] He Y, Jian T, Su F, et al. Novel range-spread target detec-tors in non-Gaussian clutter [J]. IEEE Transactions on Aerospace and Electronic Systems, 2010, 46: 1312-1328.

[13] Gerlach K. Spatially distributed target detection in non-Gaussian clutter [J]. IEEE Transactions on Aerospace and Electronic Systems, 1999, $35(3)$ : 926-934.

[14] Barton D K. Radar Systems Analysis [M]. Boston: Artech House, 1979.

[15] 简涛, 何友, 苏峰, 等. 非高斯杂波下修正的SDD距离扩展目 标检测器 [J]. 电子学报, 2009, 37(12)：2662-2667。

[16] Strong Scatterers Integrator Based on ADT in Non-Gaussian Cluter. Gu X. F., Hao X. L., Yang G. L. et. al. Science Discover, 2016, vol 4(1):26-30. 